\title{
BREEDING SYSTEMS AND HETEROZYGOSITY IN POPULATIONS OF TETRAD FORMING FUNGI
}

\author{
G. C. KIRBY \\ School of Biological Sciences, Flinders University of South Australia, Bedford Park, S.A. \\ 5042, Australia
}

Received 19.vii.83

\section{INTRODUCTION}

The breeding systems of plants and animals have been well studied and the population genetic consequences of selfing, outcrossing and asexual reproduction are understood even if the adaptive roles played by these breeding systems are still controversial (e.g., Maynard Smith, 1978). However, the breeding systems of fungi are not adequately understood. The recent analysis by Langton and Elliott (1980) of the genetic consequences of secondary homothallism in basidiomycetes shows that keeping the products of meiosis together in a tetrad (or octad) changes the expected heterozygosity in the diploid and dikaryotic progeny of a heterozygote. Langton and Elliott (1980) point out that when two products of the same meiosis pair to form a binucleate cell, sampling of genes is "without replacement" rather than "with replacement", as it is in organisms in which products of the same meiosis can rarely, if ever, come together. As a consequence, when pairing of the products of one meiosis occurs in secondarily homothallic basidiomycetes, the expected heterozygosity in the progeny is greater than 50 per cent of that in the parent, and may be as high as 66.7 per cent.

The implication of this observation for studies on fungal breeding systems is that tetrad (or octad) forming fungi can undergo self-fertilisation by two different methods:

(a) Pairing of the haploid products of different meioses on the same dikaryotic or diploid individual (intertetrad selfing);

(b) Pairing of the haploid products of the same meiosis (intratetrad selfing).

For plants and animals in which the gametes are formed in different organs in the same individual or in different individuals, the second method of selfing is not possible. The population genetic consequences of intratetrad selfing have not been worked out before and this note provides an introduction to the topic.

\section{Population genetics MOdels}

\section{(i) A single locus without mating type controls}

A simple model that ignores the effects of population subdivision, natural selection, finite population size and variability in parameter values in time and space will be introduced. The term dikaryotic will be used to describe individuals in phases of the life-cycle which may be dikaryotic or diploid. Initially, consider a tetrad forming species in which pairing of haploids is not regulated by mating type genes, but occurs at random. Denote the 
heterozygosity at a diallelic locus by $h^{i}$ in generation $i$ and the two allele frequencies by $p$ and $q$. Note that heterozygosity is assessed in the dikaryotic phase of the lifecycle.

Three kinds of mating can occur in this population:

(a) random crossing between haploid cells derived from two different randomly chosen dikaryotic individuals so that for all dikaryotic individuals formed in this way,

$$
h^{i}=2 p q
$$

(b) selfing by pairing of haploids derived from different meioses of the same dikaryotic individual (intertetrad selfing),

$$
h^{i}=\left(\frac{1}{2}\right) h^{i-1}
$$

(c) selfing by pairing of haploids derived from the same meiosis (intratetrad selfing),

$$
h^{i}=\left(\frac{2}{3}\right) h^{i-1} .
$$

The last calculation is made by observing that if we take the allele in the first of the two pairing cells as fixed, then if the parent is heterozygous, the probability that the second cell carries the same allele is only 1 in 3 and the probability that it carries the other allele is 2 in 3 because sampling is without replacement within the tetrad (Langton and Elliott, 1980).

The heterozygosity of the whole population in the next generation will be the weighted average of the contributions of each type of mating to the formation of dikaryons. Let the proportion of dikaryons formed by matings of kinds (a), (b) and (c) be $r, s$ and $t$ respectively $(r+s+t=1)$, so that,

$$
\begin{aligned}
h^{i} & =2 r p q+\left(\frac{1}{2}\right) s h^{i-1}+\left(\frac{2}{3}\right) t h^{i-1} \\
& =2 r p q+\left(s / 2+\left(\frac{2}{3}\right) t\right) h^{i-1} .
\end{aligned}
$$

If $r=0$ then $h \rightarrow 0$ as $i \rightarrow \infty$, but if $r>0$, an equilibrium between outbreeding and inbreeding can be established with equilibrium heterozygosity $(h)$ :

$$
h=\frac{2 r p q}{1-s / 2-\left(\frac{2}{3}\right) t} .
$$

The fixation index, $F$, (Wright, 1969) of the equilibrium population will be found from:

$$
F=\frac{h_{e}-h}{h_{e}}
$$

where $h_{e}$ is the expected heterozygosity under random mating $\left(h_{e}=2 p q\right)$ and $h$ (the actual heterozygosity) is found from (2).

$$
F=\frac{s / 2+t / 3}{1-s / 2-\left(\frac{2}{3}\right) t} .
$$

The effect of intratetrad selfing on the level of heterozygosity in a fungal population can be examined in two ways: firstly by examining the dynamics of the decline in heterozygosity and secondly by comparing the equilibrium levels of heterozygosity in populations in which type (c) matings are either common or rare. 
In the first case, consider two populations in which only selfing occurs, in one population via type (b) and in the other via type (c) matings. In table 1 , the decline in heterozygosity in both populations with time is shown for selected generations after both start with 50 per cent heterozygosity. The population with intratetrad selfings has 4.2 times as much heterozygosity after 5 generations and 315 times as much after 20 generations.

TABLE 1

Heterozygosity left after inbreeding by selfing in two populations: one with pairing of the products of different meioses (population 1) and the other with intratetrad pairing (population 2)

\begin{tabular}{lccccccc}
\hline & & \multicolumn{7}{c}{ Time (i) } \\
\cline { 3 - 8 } & $\begin{array}{l}\text { Recurrence } \\
\text { relationship }\end{array}$ & 0 & 1 & 2 & 5 & 10 & 20 \\
\hline Population 1 & $h^{i}=\frac{1}{2} h^{i-1}$ & 0.5 & 0.25 & 0.125 & 0.0156 & 0.00049 & 0.00000048 \\
Population 2 & $h^{i}=\frac{2}{3} h^{i-1}$ & 0.5 & 0.33 & 0.222 & 0.0658 & 0.00867 & 0.000150 \\
\hline
\end{tabular}

In the second case the advantage of intratetrad selfing under conditions where some out-crossing occurs $(r \neq 0)$ can be crudely assessed by comparing equilibrium values of $h$ for two populations:

Population 1 -all selfing by intertetrad pairing $(t=0 ; s=1-r)$;

Population 2-all selfing by intratetrad pairing $(s=0, t=1-r)$.

For population 1,

$$
\bar{h}_{1}=\frac{4 r p q}{1+r}
$$

and for population 2,

$$
\bar{h}_{2}=\frac{6 r p q}{1+2 r}
$$

The increased heterozygosity for intratetrad selfing for a given amount of outcrossing $(r)$ can be assessed by the ratio of $\bar{h}_{2}$ to $\bar{h}_{1}$ :

$$
\begin{aligned}
\text { relative heterozygosity } & =\bar{h}_{2} / \bar{h}_{1} \\
& =\frac{3(1+r)}{2(1+2 r)} .
\end{aligned}
$$

For mostly outbreeding species $(r \rightarrow 1)$ the relative heterozygosity is only slightly greater than unity but for largely selfing species ( $r$ very small), the relative heterozygosity approaches 1.5 .

(ii) Two loci, one a diallelic mating type locus (bipolar mating system)

In many fungi, the pairing of haploids (or fertility of the subsequent dikaryon) is controlled by a mating type locus with two alleles. Compatible matings occur between haploids carrying different alleles at the mating type locus (denote these alleles by $A$ and $a$ ). Any dikaryon in these species should thus be heterozygous $(A / a)$ (the cases where cell fusion between haploids with the same mating type alleles can occur will be ignored). 
The heterozygosity $(h)$ to be expected at a second diallelic locus (with alleles $B$ and $b$ ) will be considered in relation to the closeness of linkage to the mating type locus and to the centromere. Only an indication of the effects of the $A$ locus on heterozygosity at the $B$ locus will be given; a more detailed and exact account is in preparation (Barrett and Kirby in prep.).

The model must begin by considering the two principal kinds of tetrads possible from a dikaryon heterozygous for two diallelic loci:

$\begin{array}{lllll}\begin{array}{c}\text { Ditypes: } \\ \text { or }\end{array} & \frac{A B}{A b} & \frac{A B}{A b} & \frac{a b}{a B} & \frac{a b}{a B} \\ \text { Tetratype: } & \underline{A B} & \underline{A b} & \underline{a B} & \underline{a b}\end{array}$

where $\underline{A B}$ represents a haploid product of meiosis carrying alleles $A$ and $B$, and so on.

The effects of the mating type locus on the decline in heterozygosity for matings of kinds (a) and (b) will depend upon linkage and linkage disequilibrium. If there is no linkage and the coefficient of linkage disequilibrium is zero, then the mating type locus has no effect on the $B$ locus. But for the intratetrad selfing breeding system, the possible kinds of tetrads must be considered:

(i) If the tetrad is a ditype, then the assumption that only nuclei with different alleles at the mating type locus can pair implies that all dikaryons formed by intratetrad selfing must also be heterozygous at the $B$ locus. That is, heterozygosity at the $B$ locus is retained:

$$
h^{i}=h^{i-1} \text {. }
$$

(ii) If the tetrad is a tetratype, then each haploid can pair with either of two others in the tetrad, one of which carries the same $B$ allele and one of which arries the other $B$ allele. Thus the probability of a $B / b$ dikaryon is $\frac{1}{2}$.

$$
h^{i}=\frac{1}{2} h^{i-1} \text {. }
$$

If the proportion of ditype tetrads is $d$ (thus, the proportion of tetratypes is $1-d)$ then amongst the intratetrad selfings as a set,

$$
\begin{aligned}
h^{i} & =d h^{i-1}+\frac{1}{2}(1-d) h^{i-1} \\
& =\frac{1}{2}(1+d) h^{i-1} .
\end{aligned}
$$

If loci $A$ and $B$ are linked on the same chromosome, then for the case of zero interference the proportion of tetratype tetrads is given by $\frac{2}{3}\left\{1-e^{-3 x}\right\}$ (e.g. Barratt et al., 1954) where $x$ is the map length in units of $10^{-2}$ between the two loci. For very closely linked loci, $x \rightarrow 0, d \rightarrow 1$ and hence:

$$
h^{i} \rightarrow h^{i-1} \text {. }
$$

For very distant loci, $x$ becomes large, $d \rightarrow \frac{1}{3}$ and

$$
h^{i} \rightarrow \frac{2}{3} h^{i-1} \text {, }
$$

the same result as for a single locus without mating type controls.

Thus tight linkage to a mating type locus conserves heterozygosity almost absolutely for intratetrad'selfing, but the linkage can be ignored if it is very loose. 
If loci $A$ and $B$ are on different chromosomes, then the proportion of tetratype tetrads depends upon the frequency of recombination between each locus and its centromere (and hence, second division segregations). The frequency of tetratypes is $x+y-3 x y / 2$ where $x$ is the frequency of second division segregations for locus $A$ and $y$ is the same for locus $B$ (Perkins, 1949). If both loci are very closely linked to their respective centromeres, then tetratypes will be rare, $d \rightarrow 1$ and $h^{i} \rightarrow h^{i-1}$.

At the other extreme, when both loci are very distant from their centromeres, $x$ and $y$ approach $0.5, d \rightarrow \frac{3}{8}=0.375$ and $h^{i} \rightarrow \frac{11}{16} h^{i-1}=$ $0.6875 h^{i-1}$.

Conservation of heterozygosity is greater for all values of $x$ and $y$ than for the model without a mating type locus.

To summarise, the effect of the presence of a diallelic mating type locus on heterozygosity at another locus in a species in which intratetrad selfing can occur, is to decrease the rate of loss of heterozygosity in the population unless the mating type locus is very distant on the same chromosome as the locus under consideration.

A notable feature of these calculations is the difference between the case of two widely separated genes on one chromosome and the case of two genes on different chromosomes and both very distant from their centromeres. In both cases the two genes will appear unlinked genetically, but the breeding system consequences are different when intratetrad selfing occurs.

\section{Discussion}

The fungi are a large and diverse group of organisms. A common feature in the lifecycle of most fungi is the occurrence of a tetrad (or octad, as a result of an extra mitosis). This note has shown that the phenomenon of tetrad formation can increase the range of breeding system options available to fungi, relative to non-tetrad forming organisms. Self-fertilisation by the intratetrad pairing mode reduces the rate of loss of heterozygosity relative to conventional models of self-fertilisation. The exact consequences of intratetrad selfing in most fungi will depend upon the linkage relationships between the locus under study and the mating type locus (or loci). The population genetics of tetrad forming fungi will have to take account of more complexities in breeding systems than conventional models applied to animals and plants.

The significance of intratetrad selfing in the breeding system of a particular fungal species will depend upon the life history of that species. Of great importance is the dispersal stage and whether it occurs before or after tetrad formation.

A toadstool on which millions of basidia liberate haploid spores which are blown away by the wind provides a life history in which selfing, if it occurs, will be usually between the products of different tetrads. This breeding system is essentially the same as that in self-fertile plants and animals. On the other hand, a parasitic smut fungus in which the dispersal phase is a dikaryotic teliospore that germinates on contact with the host, will form a tetrad after germination. In some smuts, members of a tetrad quickly pair in mating-type compatible pairs to form infective dikaryons. 
This life-cycle greatly favours intratetrad selfings and possibly exploits the heterozygosity preserving features of intratetrad selfings.

The calculations in table 1 are also useful for designing artificial breeding programmes for fungi. If a line is being maintained by selfing then the source of gametes in each generation will need to be controlled. If an objective is to minimise the rate of inbreeding, then the products of a single meiosis should be chosen but if rapid inbreeding is required, then the products of different meioses sould be used.

The parameters $r, s$ and $t$ used to measure the proportions of dikaryons from outcrosses, intertetrad selfs and intratetrad selfs have been defined in terms of actual dikaryons formed in successful fusions. This definition ignores incompatible potential matings that occur when cells of the same mating type come into sufficiently close proximity for mating to have occurred, had the cells been of different mating type. For example, with random crossing and with intertetrad selfing when there is a diallelic mating type locus, only 50 per cent of random pairings of haploid cells will be mating type compatible. J. A. Barrett (pers comm) has pointed out that consideration of the incompatibility "load" of intratetrad selfings reveals another possible advantage of this breeding system. In a tetrad, for any haploid, two of the three remaining haploids are mating type compatible and thus 66.7 per cent of random pairings will be compatible. The incompatibility load (the propottion of random pairings that are incompatible) of outcrosses and intertetrad selfs is 50 per cent whereas that of intratetrad self is only 33.3 per cent (Barrett, pers comm).

This note is an introduction to the consequences of the intratetrad selfing mode of breeding. Considerably more research needs to be done before the topic will be fully understood. On the theoretical level, the dynamics of the two locus model need to be developed to take account of linkage disequilibrium and more complex mating type systems (e.g., 2 mating type loci with many alleles). The estimation of the parameters $r, s$ and $t$ in accessible fungal populations, and the development of models to relate high and low values of these parameters to other ecological and life history phenomena should be a long term research goal. Estimation of these parameters will not be easy as has been shown by experiments with Ustilago nuda and $U$. violacea. With $U$. nuda a loose smut in which fusion of the primary sporidia occurs soon after they are formed in a tetrad, Thomas (1978) showed that intertetrad crosses occurred. Similarly, in $U$. violacea evidence of intertetrad crosses was obtained by Baird and Garber (1979). Unfortunately, due to the deleterious nature of some of the genotypes used in these experiments, no reliable estimates could be made of $r, s$ and $t$.

Note added in proof: An independent derivation of some of the results in this note has been located (Zakharov, 1968).

Acknowledgements. I am grateful to F. A. Langton and J. A. Barrett for correcting several errors in a draft of this note. This research was supported by the A.R.G.S. and The Flinders University Research Budget.

\section{REFERENCES}

BAIRD, M. L. AND GARBER, E. D. 1979. Genetics of Ustilago violacea. V. Outcrossing and selfing in teliospore inocula. Bot. Gaz., 140, 89-93. 
BARRATT, R. W., NEWMEYER, D., PERKINS, D. D. AND GARNJOBST, L. 1954. Map construction in Neurospora crassa. Advances in Genetics 6, 1-92.

LANGTON, F. A. AND ELLIOTT, T. J. 1980. Genetics of secondarily homothallic basidiomycetes. Heredity, 45, 99-106.

MAYNARD SMITH, J. 1978. The Evolution of Sex. Cambridge University Press, Cambridge. PERKINS, D. D., 1949. Biochemical mutants in the smut fangus Ustilago maydis. Genetics, 34, 607-626.

THOMAS, P. L. 1978. Potential for natural hybridisation between strains of Ustilago nuda Can. J. Bot., 56, 721-726.

WRIGHT, S. 1969. Evolution and the Genetics of Populations, Volume 2. The theory of Gene Frequencies. University of Chicago Press, Chicago.

ZAKHAROV, I. A. 1968. Homozygosity in intratetrad and intraoctad fertilisation in fungi. Genetica, 4, 18-105. (Translated in Soviet Genetics, 4, 636-642). 